\title{
Implementation of a Low-Cost Energy and Environment Monitoring System Based on a Hybrid Wireless Sensor Network
}

\author{
Dong Sik Kim, ${ }^{1}$ Beom Jin Chung, ${ }^{2}$ and Sung-Yong Son ${ }^{3}$ \\ ${ }^{1}$ Department of Electronics Engineering, Hankuk University of Foreign Studies, Yongin-si, Gyeonggi-do 17035, Republic of Korea \\ ${ }^{2}$ Smart Green Home Research Center, Gachon University, Gyeonggi-do, Republic of Korea \\ ${ }^{3}$ Department of Electrical Engineering, Gachon University, Gyeonggi-do, Republic of Korea \\ Correspondence should be addressed to Sung-Yong Son; xtra@gachon.ac.kr
}

Received 17 March 2017; Revised 19 June 2017; Accepted 28 June 2017; Published 31 July 2017

Academic Editor: Stefania Campopiano

Copyright (C) 2017 Dong Sik Kim et al. This is an open access article distributed under the Creative Commons Attribution License, which permits unrestricted use, distribution, and reproduction in any medium, provided the original work is properly cited.

\begin{abstract}
A low-cost hybrid wireless sensor network (WSN) that utilizes the $917 \mathrm{MHz}$ band Wireless Smart Utility Network (Wi-SUN) and a $447 \mathrm{MHz}$ band narrow bandwidth communication network is implemented for electric metering and room temperature, humidity, and $\mathrm{CO}_{2}$ gas measurements. A mesh network connection that is commonly utilized for the Internet of Things (IoT) is used for the Wi-SUN under the Contiki OS, and a star connection is used for the narrow bandwidth network. Both a duty-cycling receiver algorithm and a digitally controlled temperature-compensated crystal oscillator algorithm for frequency reference are implemented at the physical layer of the receiver to accomplish low-power and low-cost wireless sensor node design. A two-level temperaturecompensation approach, in which first a fixed third-order curve and then a sample-based first-order curve are applied, is proposed using a conventional AT-cut quartz crystal resonator. The developed WSN is installed in a home and provides reliable data collection with low construction complexity and power consumption.
\end{abstract}

\section{Introduction}

Indoor temperatures are traditionally controlled using thermostats and their corresponding actuators or valves, as shown in Figure 1(a). In order to sense the room temperatures of everyday living spaces efficiently, wireless sensors utilizing the industrial, scientific, and medical (ISM) $2.4 \mathrm{GHz}$ radio band with wireless link distances that are usually restricted within a single room, as shown in Figure 1(b), have recently been developed.

It is essential to monitor the indoor air quality of residential and commercial apartment complex areas efficiently based on measured room temperatures, humidity levels, $\mathrm{CO}_{2}$ gas amounts, and other factors to control the indoor environmental conditions properly. A particular monitoring system usually depends on the existing building automation system (BAS), as shown in Figure 2, as well as on the data collected by the numerous sensors in the building and stored in a BAS server. The exploitation of wireless sensor network (WSN) technology can provide further efficiency and flexibility in data collection approaches $[1,2]$. Energy usage data, such as those collected from electric metering in the advanced metering infrastructure (AMI), can also be collected efficiently through collaboration with environment data collection. Furthermore, a WSN can deliver energy information to the in-home display (IHD) system efficiently [3]. The sensor node, which is considered in this paper, can deliver electrical energy usage information and measure temperature and humidity values. The proposed WSN can transfer and collect both energy and environment data.

For mass deployment in residential spaces or office rooms, both the construction cost and the complexity of a WSN should be minimized while still maintaining reliable wireless link margins $[4,5]$. Based on an Internet of Things (IoT) technique using IPv6, we can construct a WSN. However, instead of implementing the IoT WSN for all sensors, including the end nodes, which could result in a high cost, partially adopting the IoT WSN can reduce the implementation cost and decrease the power consumption. In order to build a low-cost IoT-based WSN, a hybrid 


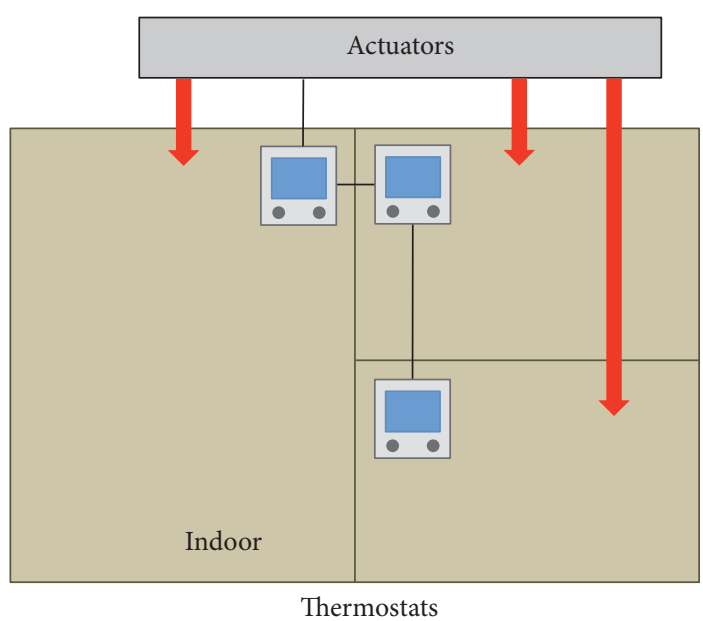

(a)

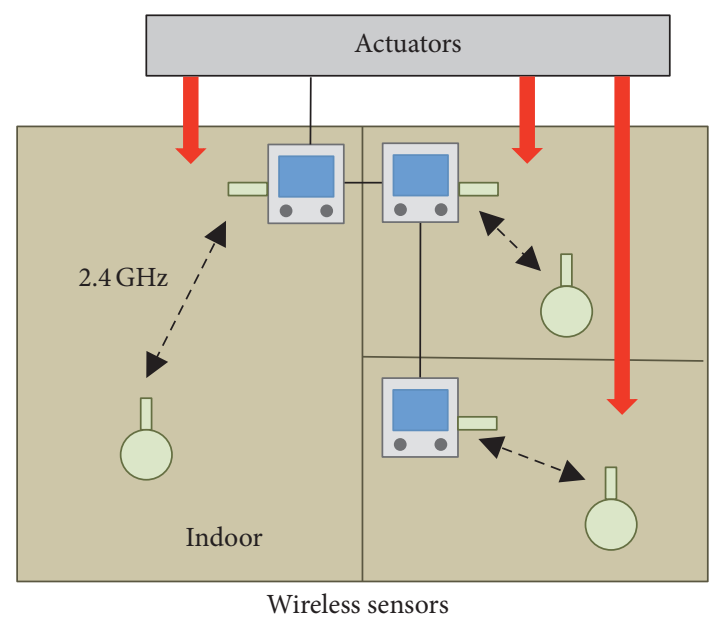

(b)

FIGURE 1: Indoor temperature control based on temperature sensors. (a) Traditional thermostats and actuators. (b) Wireless sensors for accurate temperature sensing.

WSN that exploits both high and low data-rate bands is developed [6]. In the hybrid WSN, the high-rate wireless link controls a mesh network in order to collect and transmit data to a server based on the IoT, and the low-rate wireless link collects data directly through a star-connected narrow bandwidth (NB) communication channel at low data rates in order to guarantee long-lasting wireless links [7]. The Korean regulations for $\mathrm{NB}$ radio stipulate an $8.5 \mathrm{kHz}$ bandwidth with $12.5 \mathrm{kHz}$ channel spacing, while the high-rate wireless link can have a much broader bandwidth.

In this paper, a low-cost hybrid WSN system utilizing the $917 \mathrm{MHz}$ and $447 \mathrm{MHz}$ radio bands is developed and implemented for energy and environmental monitoring [8]. The RFID/USN, or Wi-SUN (IEEE802.15.4 g), $917 \mathrm{MHz}$ radio band with $200 \mathrm{kHz}$ channel spacing is used to achieve a more stable RF link margin than the one that the $2.4 \mathrm{GHz}$-ISM band can achieve. In apartment and home environments, transmitted RF signals can reach other rooms due to diffraction of the signals. Hence, a lower frequency of $917 \mathrm{MHz}$ is advantageous to a $2.4 \mathrm{GHz}$ frequency for a high data-rate network $[7,9]$. In a low data-rate network, the use of NB radios utilizing the $447 \mathrm{MHz}$ band can significantly reduce the number of gateways. In order to achieve both low-power and low-cost goals, a duty-cycling algorithm [10] is developed at the physical layer of the receiver. Also, a digitally controlled temperature-compensated crystal oscillator (DCTCXO) [11] in which a low-cost microcontroller unit (MCU) controls the oscillation frequency by utilizing several functions of the RF integrated circuit (RFIC) is developed.

This paper is organized as follows. In Section 2, the architecture of the proposed low-cost hybrid WSN is described. In Section 3, an implementation of the network in residential indoor environment monitoring is introduced. In Section 4, a low-cost implementation of an RF radio component containing a DCTCXO is discussed. The paper is concluded in the final section.

\section{The Low-Cost Hybrid Wireless Sensor Network}

In this section, a low-cost implementation of the hybrid WSN for indoor environment monitoring is introduced.

The IoT technique, which utilizes Wi-Fi or Wi-SUN, can be employed to gather the indoor temperature data for temperature control. The distance ranges for most commonly used Wi-Fi (IEEE802.11) are 20-70 meters and 100-250 meters for indoor and outdoor environments, respectively. Hence, each house should have an individual IoT gateway, as shown in Figure 3. The sub-1 GHz Wi-SUN band can also implement IoT-based sensors, and each gateway can collect more data from several houses than it can in the Wi-Fi case because of the narrower receiver bandwidth and better diffraction properties. The wider collection capability of WiSUN can reduce the implementation cost when compared to that of the Wi-Fi case. However, a much lower implementation cost is required for practical residential environment monitoring.

As illustrated in Figure 2, a $447 \mathrm{MHz}$ band $\mathrm{NB}$ radio can be used in a customized network to gather sensed data. In this case, a simple gateway can collect the data from numerous sensors in several houses similarly to the way it can in a Wi-SUN implementation, as shown in Figure 4. However, because the customized network is simpler than the network used in the Wi-SUN case, the installation cost of the WSN, which is based on the use of NB radios in a customized network, is significantly lower than the costs of both the WiFi and Wi-SUN installations. The simple gateway in Figure 4 can be connected to Ethernet through a Wi-Fi interface in order to transmit the collected data to a data server.

If a small number of sensors are scattered over a wide area (e.g., over a whole building), then the LoRa modulation technology is an appropriate candidate for NB radio because of its long-range capability and its low data rate of $18 \mathrm{bps}$ 


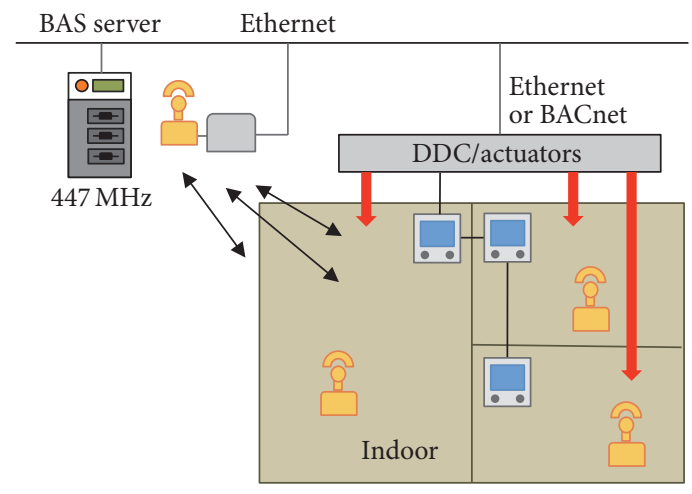

Wireless sensors

Figure 2: Indoor temperature control based on both the building automation system (BAS) and wireless sensors.

operating at a $7.8 \mathrm{kHz}$ receiver bandwidth, as shown in Figure 4. However, assuming that the number of sensors is large, collecting all of the data from a single gateway under the very low data rate of LoRa technology requires a considerably long time. In order to alleviate the low datarate problem occurring in $\mathrm{NB}$ radios while achieving a long wireless distance, a hybrid WSN system [6], as illustrated in Figure 5, is employed in this study. To collect the sensed data efficiently, the hybrid WSN includes both a low datarate network based on a customized star connection and a high data-rate network based on a standard mesh connection. The low data-rate network is constructed using NB radios to ensure that the RF link has a long range because it is generally believed to minimize transmission power and protocol while achieving a very low implementation cost. On the other hand, the high data-rate network collects sensed data from the sensors corresponding to the low data-rate star connection, gathering them through the high data-rate mesh connection, to send them to a server. The power-consumption constraint is not as significant for the sensor nodes in the high data-rate network as it is for those in a low data-rate one.

\section{Implementation of the Hybrid Wireless Sensor Network}

In this section, an implementation of the hybrid WSN in indoor environment monitoring is introduced. In the implemented network, the low data-rate network transmits sensed data periodically through the NB radios under a nonslotted ALOHA protocol. On the other hand, the high data-rate network operates through radios with relatively wider bandwidths under a constrained application protocol (CoAP) using the Contiki OS.

Figure 5 shows an overview of the implemented hybrid WSN system in which three types of components are used: simple wireless sensors, CoAP wireless sensors, and a CoAP gateway. Simple wireless sensors can measure room temperature and humidity without consuming much power and can transmit the sensed data to the corresponding collector, the CoAP wireless sensor, through an NB air link utilizing the $447 \mathrm{MHz}$ band. This process corresponds to that of the

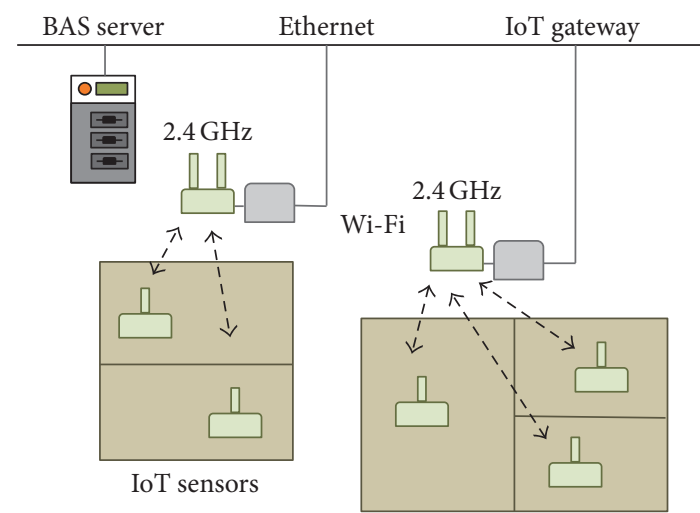

FIGURE 3: Indoor environment monitoring based on the Internet of Things (IoT).

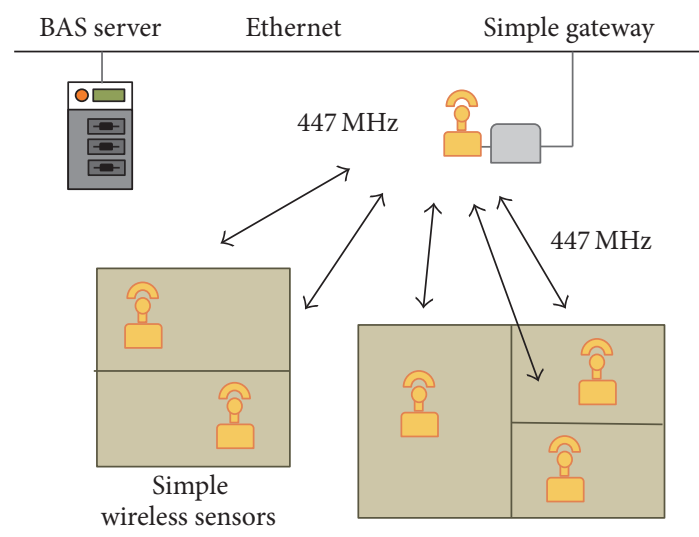

FIGURE 4: Indoor environment monitoring based on the use of NB radio modules under the $447 \mathrm{MHz}$ band.

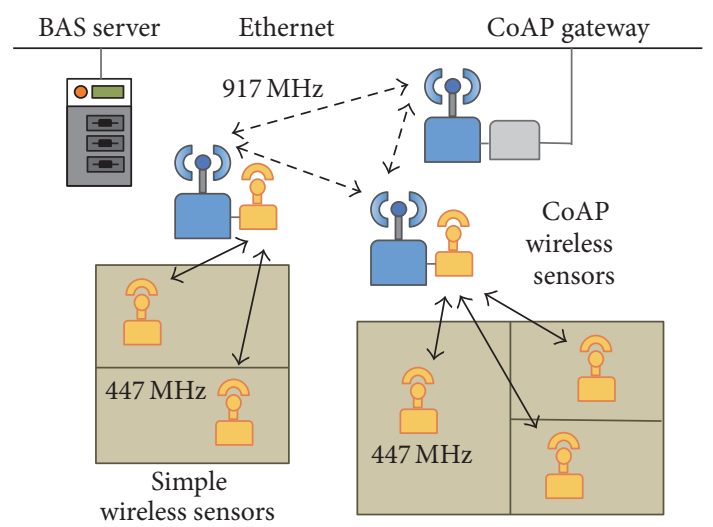

FIGURE 5: Implemented indoor environment monitoring system based on the use of the hybrid WSN under the $917 \mathrm{MHz}$ and $447 \mathrm{MHz}$ bands.

low data-rate star connection in the hybrid WSN. A mesh network utilizing a $917 \mathrm{MHz}$ band $\mathrm{RF}$ link under the routing protocol for low-power and lossy networks (RPL) as well as the low-power IPv6 network (6LowPAN) protocol using the Contiki OS is constructed among the CoAP wireless sensors. The CoAP wireless sensors (CoAP server) can measure the 


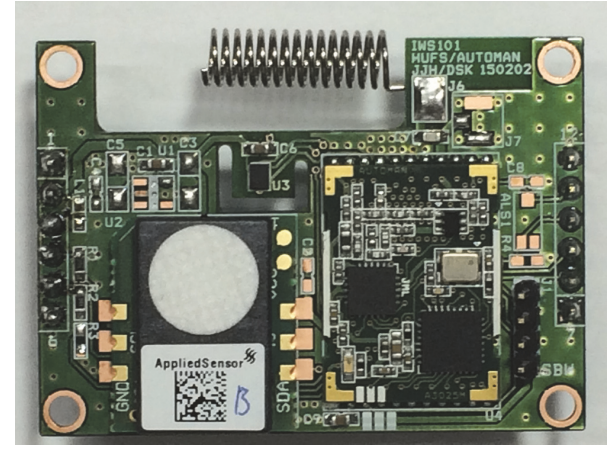

FIGURE 6: Integrated wireless sensor module (IWSM) containing a $447 \mathrm{MHz}$ band RF module with a helical antenna, temperature and humidity sensor, and optional $\mathrm{CO}_{2}$ sensor.

amount of present $\mathrm{CO}_{2}$ gas as well as the local temperature and humidity. The CoAP gateway (CoAP client), which is an Ethernet-based mesh network gateway, transmits collected data to the BAS server through Ethernet under the CoAP RESTful application-layer protocol.

In order to manufacture the hybrid WSN system efficiently, an integrated wireless sensor module (IWSM) is developed, as shown in Figure 6. The IWSM contains an $\mathrm{RF}$ radio module, which is composed of a low-cost MCU (TI, MSP430G2553), an RFIC (Silicon Labs, Si4463) with a frequency reference, and a temperature and humidity sensor. The option of adding a $\mathrm{CO}_{2}$ gas sensor is available. The $\mathrm{CO} 2$ sensor, which is based on the metal-oxide-semiconductor (MOS) technology (IAQ-CORE), is employed for low-power consumption with a small size instead of the conventional nondispersive infrared (NDIR) type sensors. The NDIRbased $\mathrm{CO}_{2}$ sensor can usually provide accurate measurements of the $\mathrm{CO}_{2}$ gas amount. However, the sensor size is relatively big and requires high powers with a long standby time. On the other hand, the MOS-based $\mathrm{CO}_{2}$ sensor can efficiently measure the $\mathrm{CO}_{2}$ gas amount in an indirect method with low-power consumption. The low-cost MCU has a flash memory of $16 \mathrm{kB}$ and a RAM of $512 \mathrm{~B}$ but does not have any hardware multiplier. In the standby and active modes, the MCU consumes currents of $0.5 \mu \mathrm{A}$ and $330 \mu \mathrm{A}$, respectively, at an operating voltage of $3.0 \mathrm{~V}$. An NB radio for use in an industrial temperature range from $-40^{\circ} \mathrm{C}$ to $85^{\circ} \mathrm{C}$ requires a temperature-compensated crystal oscillator (TCXO) component with a frequency error of $\pm 2 \mathrm{ppm}$ to ensure communication. Even though the present study only requires sensing indoor temperatures in commercial temperature range of $0^{\circ} \mathrm{C}-70^{\circ} \mathrm{C}$, the industrial specification is applied to the NB radio design. Operated at a data rate of $2.4 \mathrm{kbps}$ with an $8.5 \mathrm{kHz}$ receiver bandwidth and a modulation index of 0.5 based on GFSK modulation, the receiver sensitivity of the proposed IWSM design is $-118 \mathrm{dBm}$ with a bit error rate less than $0.1 \%$ and a transmission power of $10 \mathrm{dBm}$. The typical accuracies of temperature and humidity sensors are $\pm 0.3^{\circ} \mathrm{C}$ and $\pm 2 \% \mathrm{RH}$, respectively.

As shown in Figure 7(a), the simple wireless sensor is composed of an IWSM without a $\mathrm{CO}_{2}$ sensor and is powered by two "AA" batteries for ease of installation and maintenance in residential areas. Hence, both its power consumption and implementation cost should be very low as mentioned in the properties of the low-cost hybrid WSN. A lithium iron disulfide $\left(\mathrm{Li}-\mathrm{FeS}_{2}\right)$ battery can be used to sense outdoor temperatures as low as $-40^{\circ} \mathrm{C}$. The sensors in each room of several houses can transmit measured data to the corresponding CoAP wireless sensors via star connections at the same low data rates as those of $447 \mathrm{MHz}$ band $\mathrm{NB}$ communications.

The CoAP wireless sensor, which is based on a CortexM3 processor, collects measured data from simple wireless sensors through the $447 \mathrm{MHz}$ band RF module of the IWSM. Then, it shares the data with other CoAP wireless sensors and with the CoAP gateway via a mesh wireless network utilizing the IPv6 network protocol under both the Contiki OS [12] and CoAP through the $917 \mathrm{MHz}$ band RF module, as shown in Figure 7(b). The CoAP sensor uses AC power and can measure the amount of $\mathrm{CO}_{2}$ gas present. The CoAP gateway contains a Cortex-A8 platform (Beaglebone Black) to communicate through Ethernet with the BAS server under the Linux OS, as shown in Figure 8. The gateway also forms a mesh network with the CoAP wireless sensors under the $917 \mathrm{MHz}$ band. For a network with ten CoAP wireless sensors and a CoAP gateway, the average transmission delay time was $13.8 \mathrm{~ms}$ at a transmission rate of $99.9 \%$.

\section{Low-Power and Low-Cost Wireless Sensors}

For a low-cost implementation of the simple wireless sensor with low-power consumption, the RF module should consume little power in both receiving and transmitting modes. A superregenerative receiver can significantly reduce both the power consumption and implementation cost under a low operating voltage [13]. In this paper, the superheterodyne receiver of a commercially available RFIC is used to achieve a longer range than that of a superregenerative receiver. In order to reduce the power consumption of the RFIC, a duty-cycling algorithm is employed based on a fast direct preamble detection algorithm. The DCTCXO algorithm is then implemented.

4.1. Duty-Cycling Receiver Algorithm. In order to reduce the average power consumption of the RF module receiver, an efficient duty-cycling algorithm is employed in which the receiver is turned on and off periodically to detect the transmitted preamble signal. Here, the duty cycle is proportional to the average operating current of the receiver. The receiver checks the existence of the preamble signal over a short period of activity. If the preamble signal is detected, then the receiver remains on until the reception of the entire data packet is complete. If there is no preamble signal, the receiver is turned off immediately. In order to reduce the average operating current, a fast detection scheme is developed to decrease the preamble detection time. In the scheme, the MCU of the RF module observes each directly received signal to verify whether the signal is correct or not while decoding the packet. Without a duty-cycling algorithm, the average current is $16.7 \mathrm{~mA}$, which can be reduced to 


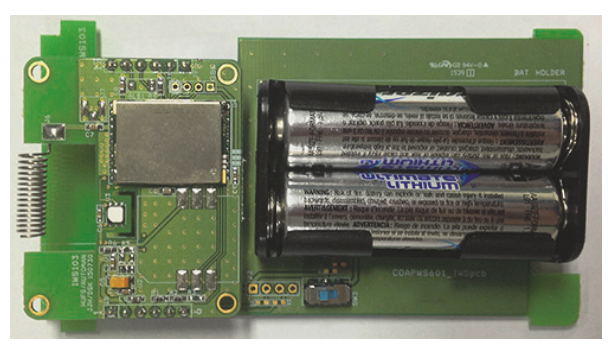

(a)

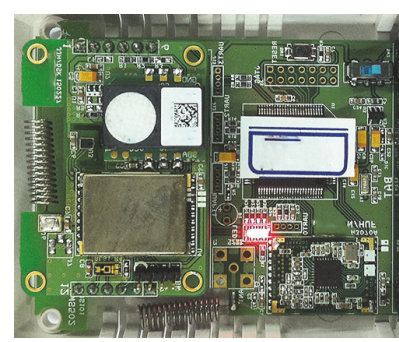

(b)

FIGURE 7: Simple and CoAP wireless sensors. (a) Simple wireless sensor circuit board. (b) CoAP wireless sensor circuit board.

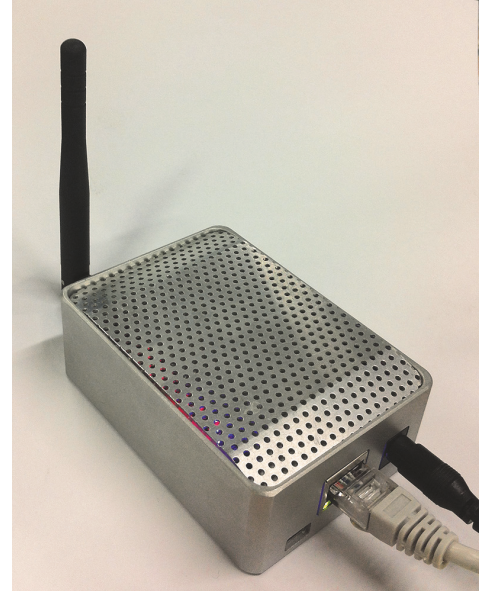

(a)

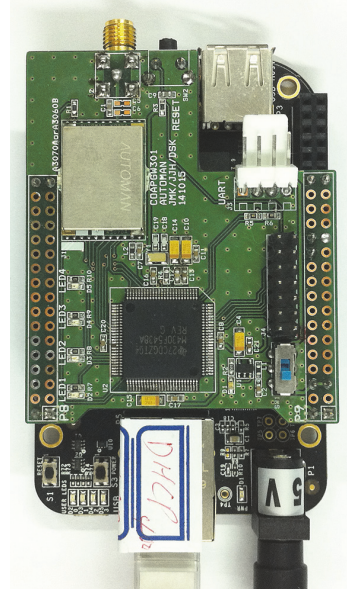

(b)

Figure 8: CoAP gateway. (a) Exterior of CoAP gateway. (b) CoAP gateway circuit board with a $917 \mathrm{MHz}$ band RF module and a Cortex-A8 processor.

$10.4 \mathrm{~mA}$ using a conventional duty-cycling algorithm with synchronized detection. However, the average current can be further reduced to $5.6 \mathrm{~mA}$ by applying the proposed dutycycling algorithm based on fast preamble detection.

\subsection{Digitally Controlled Temperature-Compensated Crystal} Oscillator. In order to obtain a sufficient RF link margin, an NB RF module with a TCXO component can be employed, as shown in Figure 9(a). The operating current of the TCXO component is $1.8 \mathrm{~mA}$. Therefore, using a conventional crystal resonator instead of the TCXO component can reduce the average operating currents in both the receiver and transmitter modes. Furthermore, replacing the expensive TCXO component with a conventional crystal resonator can significantly reduce the implementation cost. However, the frequency error, which depends on variations in temperature, increases the receiver bandwidth, and thus both the receiver sensitivity and selectivity are degraded, even though the automatic frequency control (AFC) function is active. For a crystal resonator with a frequency error of $\pm 20 \mathrm{ppm}$, the receiver bandwidth is $36.6 \mathrm{kHz}$. Hence, the receiver is highly susceptible to adjacent channel transmissions under $12.5 \mathrm{kHz}$ channel spacing. On the other hand, the frequency error of the TCXO shown in Figure 9 (a) is \pm 2.0 ppm with a receiver bandwidth of $6.5 \mathrm{kHz}$. Narrowing the bandwidth increases the receiver sensitivity, and thus the transmission power can be reduced while still achieving a similar RF link margin. Consequently, the power consumption can be reduced [12, 13].

In this study, in order to achieve a similar receiver sensitivity performance to that of the TCXO with a frequency error of $\pm 2.0 \mathrm{ppm}$, a DCTCXO algorithm is implemented based on a digital technique using the inherent functions of both the MCU and RFIC of the RF module for the AT-cut quartz crystal resonator, as shown in Figure 9(b). Digital techniques have been employed to design higher-precision TCXO than that which analog techniques are able to achieve. Buroker and Frerking [11] and Mroch and Hykes [14] used the available memory, the digital-to-analog converters (DACs), and the varactor diodes to compensate the crystal frequencies with both coarse analog and fine digital techniques with respect to temperature. Azcondo et al. [15] proposed microcomputer temperature compensation of a crystal oscillator by utilizing both the dual mode operation and the varactor diodes. The 


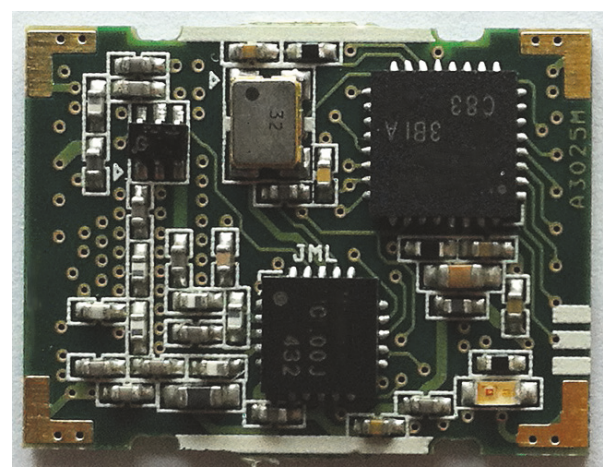

(a)

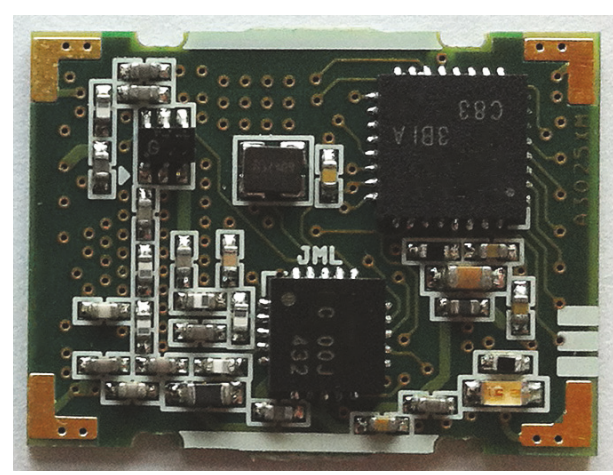

(b)

FIGURE 9: Digitally controlled temperature-compensated crystal oscillator (DCTCXO). (a) NB RF module with a TCXO component $( \pm 2.0 \mathrm{ppm})$. (b) NB RF module with both an AT-cut quartz crystal resonator $( \pm 20 \mathrm{ppm})$ and a DCTCXO algorithm implementation for a $\pm 2.0 \mathrm{ppm}$ frequency error.

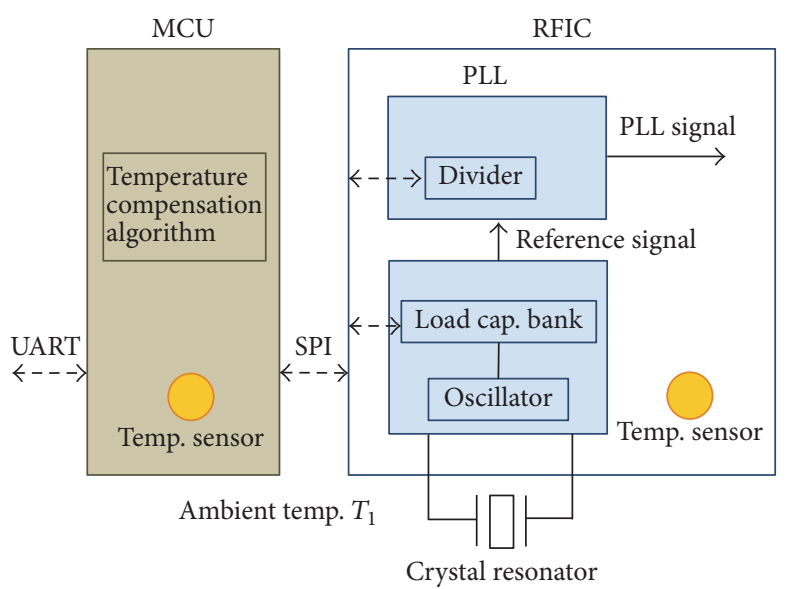

FIGURE 10: Frequency control portion of the RF module with the AT-cut quartz crystal resonator in the proposed DCTCXO.

capacitor banks were also used to trim the crystal frequency $[16,17]$. The purpose of employing the digital technique, which is introduced to the proposed DCTCXO, is to achieve the same performance as that of the analog TCXO while minimizing the production procedure by not adding any components.

A block diagram of the frequency control portion of the designed DCTCXO is illustrated in Figure 10. Instead of using the DACs and the varactor diodes, the crystal oscillation frequency can be easily controlled by the load capacitor bank, which can be selected by the MCU. From an externally connected frequency counter, the channel frequency $f_{0}$ is tuned to a temperature of $25^{\circ} \mathrm{C}$ by adjusting the RFIC load capacitors to provide a coarse resolution of $400 \mathrm{~Hz} / \mathrm{div}$ (<1 ppm trimmable). The phase locked loop (PLL) divider register values are then calculated from the measured temperatures with a fine resolution of $15 \mathrm{~Hz} / \mathrm{div}$ to compensate the crystal frequency for temperature. Here, the crystal's temperature can be measured by the temperature sensor of either the MCU or the RFIC. The accuracy of the temperature sensors operating within the temperature range from $-40^{\circ} \mathrm{C}$ to $85^{\circ} \mathrm{C}$ is usually as low as $\pm 2^{\circ} \mathrm{C}$. Furthermore, because of the heat that emanates from the RFIC when it operates in either receiver or transmitter mode, measurement of the crystal's temperature results in transient responses with biases [17]. Hence, the measured temperatures should be compensated for according to the mode in which the RF is operating.

The proposed DCTCXO algorithm is now introduced. For the channel frequency $f_{0}$, the relative frequency change of the AT-cut quartz crystal resonator can be represented by a third-order curve, where the frequency deviation at the inflection temperature $T_{0}$ is zero, as follows:

$$
\frac{\Delta f}{f_{0}}=A_{1}\left(T-T_{0}\right)+A_{2}\left(T-T_{0}\right)^{2}+A_{3}\left(T-T_{0}\right)^{3},
$$

where the numerator $\Delta f:=f-f_{0}$ is the frequency change. In (1), the parameters $A_{1}, A_{2}$, and $A_{3}$ are constants that depend on the physical properties of the crystal unit, including the angle of its cut, ratios of its dimensions, and order of its overtone. The inflection temperature of the AT-cut crystal is 
approximately equal to $25^{\circ} \mathrm{C}$. If we can obtain the parameters for a set of crystal resonators, then we can compensate the crystal frequency for temperature by using the temperature curve in (1). However, the parameters of the crystal resonators can vary during manufacturing due to nonuniform cut angles, and these parameters are determined by taking samples. Hence, the accuracy of temperature compensation performed by using a single compensation curve with fixed parameters can exhibit errors that are quite large. Therefore, a simple technique for measuring the nonuniformity of a crystal resonator sample is necessary to conduct temperature compensation more accurately. Let $\Delta \theta:=\theta-\theta_{0}$ denote the cut-angle difference between the intended angle $\theta$ and the zero-temperature coefficient-reference angle $\theta_{0}$, measured in degrees. For the AT-cut crystal resonator, Bechmann [18] found a change rate of $-0.08583 \times 10^{-6} \Delta \theta$ for the first parameter $A_{1}$ with respect to the cut-angle change $\Delta \theta$. In contrast, $A_{2}$ and $A_{3}$ had relatively lower change rates of $-0.07833 \times 10^{-9} \Delta \theta$ and $-0.033 \times 10^{-12} \Delta \theta$, respectively. Hence, the cut-angle change $\Delta \theta$ dominates the value of $A_{1}$ but not the values of $A_{2}$ and $A_{3}$. In other words, various crystal resonator samples with different values of $\Delta \theta$ can have approximate third-order curves of (1) in which the values of $A_{1}$ vary, while $A_{2}$ and $A_{3}$ do not change significantly. Therefore, the difference between the two third-order curves of (1) can be approximated by a first-order curve. After applying temperature compensation using a fixed third-order curve of (1), the relative change of the resultant frequency $f^{\prime}$ can be given as a first-order curve that is equal to zero at the inflection temperature $25^{\circ} \mathrm{C}$, as

$$
\frac{\Delta f^{\prime}}{f_{0}} \approx a_{1}\left(T-T_{0}\right),
$$

where the constant $a_{1}$ is defined as $a_{1}:=A_{1}-A_{1}^{\prime}$ and $A_{1}^{\prime}$ is the first parameter of the crystal resonator sample to be temperature compensated. In (2), the numerator $\Delta f^{\prime}:=$ $f^{\prime}-f_{0}$ is the resultant frequency change. By measuring the parameter $a_{1}$ in (2), the third-order compensated frequency curve can be compensated once again to achieve an even more accurate frequency reference. In the proposed DCTCXO, the calculation of $a_{1}$, which depends on the crystal resonator sample, is automatically performed using the frequency-offset measurement ability of the RFIC's AFC block. As shown in Figure 11, a reference-frequency RF signal originating from a high-precision RF signal generator enters the RF module, and $\Delta f^{\prime}$, the frequency offset of the crystal resonator sample at an ambient temperature of $T_{1}$, which is different for $25^{\circ} \mathrm{C}$, is calculated by the AFC. The parameter $a_{1}$ can then be obtained from the frequency-offset register of the AFC. The proposed DCTCXO algorithm can be summarized as follows.

4.2.1. DCTCXO Algorithm. First, conduct third-order temperature compensation using a fixed third-order curve of (1). Second, conduct first-order temperature compensation using the first-order curve of (2), which is obtained from the crystal resonator sample to be temperature compensated.

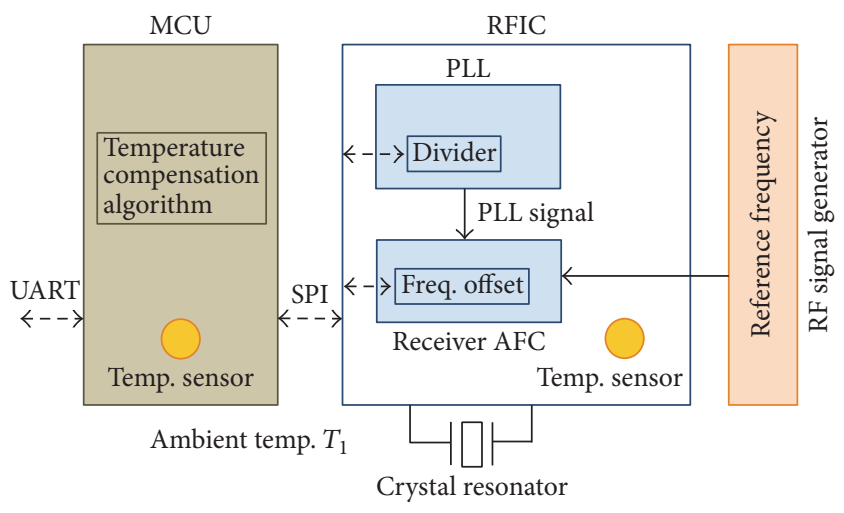

FIGURE 11: Frequency-offset measurement portion of the RF module for first-order compensation in the proposed DCTCXO.

A more detailed description of the algorithm and practical experimental observations will be introduced in the results section.

\section{Experimental Results}

In this section, an experiment performed on the proposed DCTCXO is introduced first. Then, a low-cost hybrid WSN, constructed with a low-cost RF module and implemented in a home, is introduced.

5.1. Proposed DCTCXO for the Low-Cost RF Module. The relative frequency changes versus the environment temperatures of five RF module samples were obtained experimentally, as shown in Figure 9(b). AT-cut crystal resonators were used to generate the RF reference frequency of $32.0000 \mathrm{MHz}$. In Figure 12(a), the frequency errors of the five RF module samples, given in parts per million (ppm), are shown and fitted to third-order curves. Using the fitted third-order curves, the results of temperature compensation performed on the five samples are shown in Figure 12(b). The compensation reduced the maximum frequency error from $\pm 9.3 \mathrm{ppm}$ to $\pm 2.8 \mathrm{ppm}$. As addressed in (2), the compensated curves are first-order curves whose shapes depended on the crystal resonator samples. As shown in Figure 13(a), first-order fitting was conducted on each compensated result appearing in Figure 12(b). The second iteration of temperature compensation was then performed using the first-order curve corresponding to each crystal resonator sample. The final compensated curves are shown in Figure 13(b), revealing that the maximum frequency error was reduced significantly to $\pm 0.68 \mathrm{ppm}$.

Unfortunately, first-order fitting using all the crystal sample data, shown in Figure 13, is not practical. Hence, a simple, practical technique, in which a frequency-offset measuring technique was employed by the RFIC's AFC block, was used to find the first-order curve of the crystal sample, as shown in Figure 11. In Figure 14, the frequencies measured using the frequency-offset register of the AFC are depicted versus the true frequency offsets. Biases existed depending on the input frequency offsets, and a first-order curve $\eta$ was 


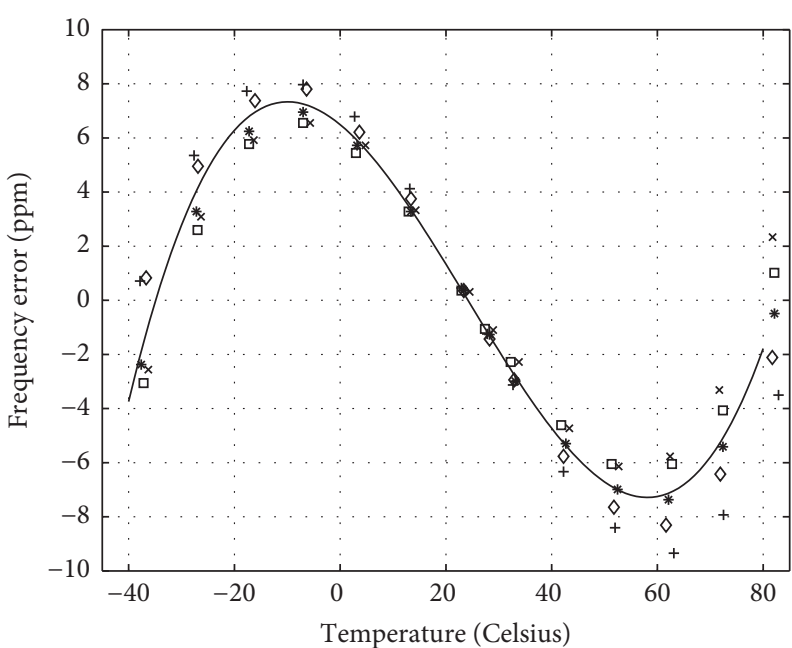

- Third-order curve

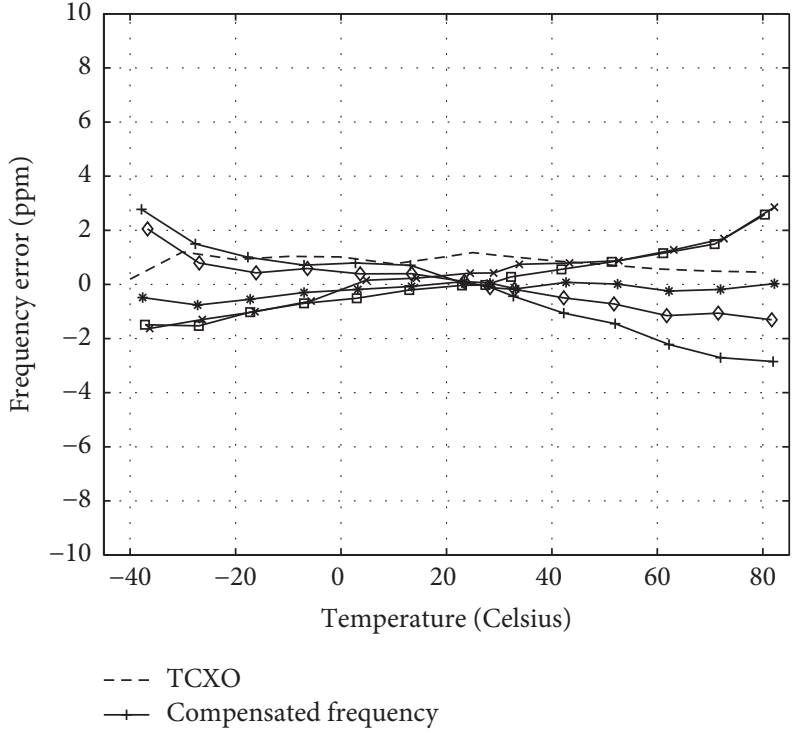

(b)

FIGURE 12: Temperature compensation using a fixed third-order curve. (a) Frequency errors (in ppm) versus ambient temperatures with third-order curves fitted to the five RF model samples ( $\pm 9.3 \mathrm{ppm})$. (b) Temperature-compensated results using the fitted third-order curve $( \pm 2.8 \mathrm{ppm})$.

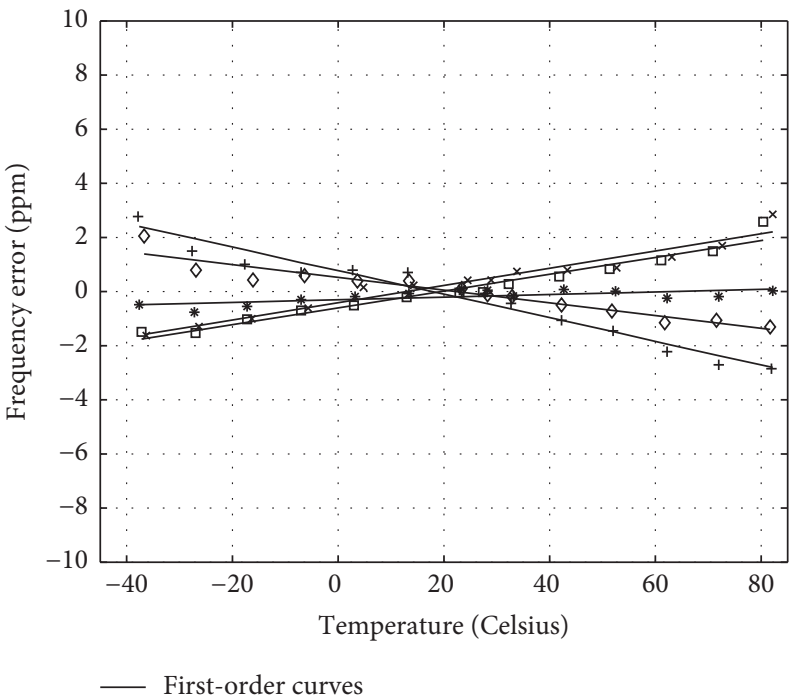

(a)

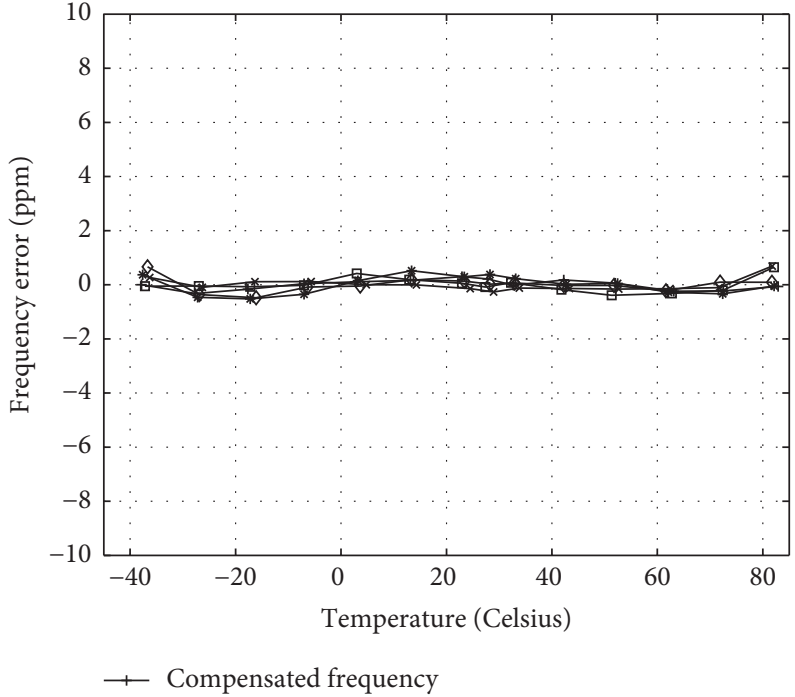

(b)

FIGURE 13: Temperature compensation based first on the fixed third-order curves and then on the individual first-order curves. (a) First-order curve fitting for each RF module sample using all of the third-order compensated data of Figure 12(b). (b) Temperature-compensated results using first the fixed third-order curves and then the individual first-order curves $( \pm 0.68 \mathrm{ppm})$.

used to describe the bias curve. Hence, in order to use the frequency $f_{\text {offset }}$ measured from the frequency-offset register of the AFC at a specific ambient temperature $T_{1}$ that is higher than the inflection temperature $T_{0}$, compensation using the inverse function of the bias curve $\eta^{-1}$ should be applied. A curve of (2) obtained this way is then given by the parameter $a_{1}$ as

$$
a_{1}=\frac{\eta^{-1}\left(f_{\text {offset }}\right)}{T_{1}-T_{0}} .
$$

The first-order curves obtained from both the frequencyoffset registers and (3) are depicted in Figure 15(a), and 


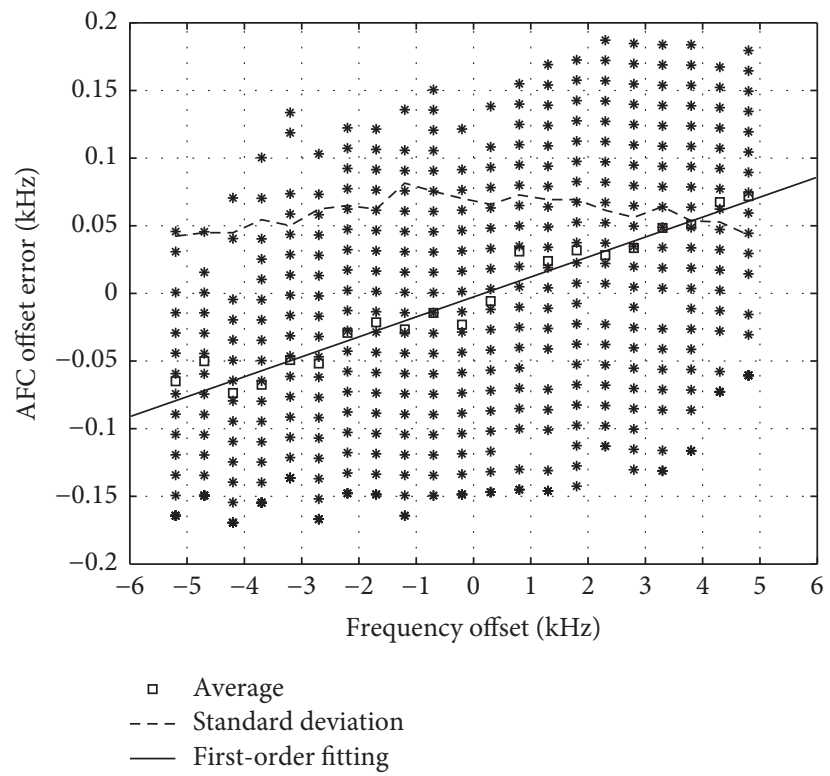

FIGURE 14: Frequency-offset measurement from the AFC frequency-offset register of RFIC. The compensation curve is obtained by fitting a first-order curve to the measured frequency offsets from the frequency-offset register of AFC.

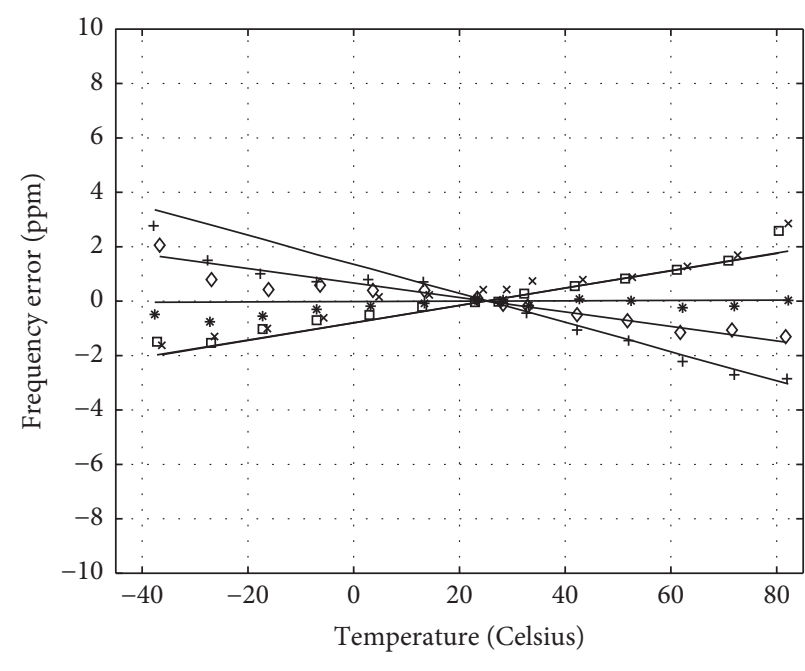

- First-order curves

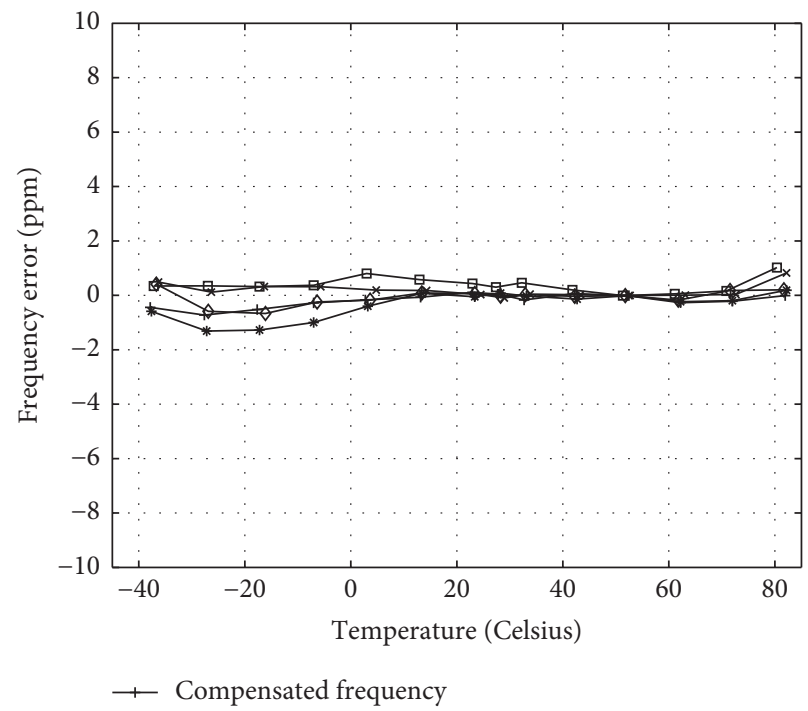

(b)

FIGURE 15: Temperature compensation in the proposed DCTCXO. (a) First-order curve fitting for each RF model sample using (3) at $50^{\circ} \mathrm{C}$. (b) Temperature-compensation results using the fixed third-order curve and then the individual first-order curve $( \pm 1.3 \mathrm{ppm})$.

the compensated results are shown in Figure 15(b). The fitting increased the maximum frequency error slightly to \pm 1.3 ppm from that of Figure 13(a). In the experiment shown in Figure 15, the ambient temperature was $T_{1} \approx 50^{\circ} \mathrm{C}$. For a practical RF module design, it is important to measure one frequency error at a specific temperature and to conduct automatic compensation, even though the temperaturecompensation performance is degraded slightly, especially at a low temperature range. The memory required to implement the proposed DCTCXO was $520 \mathrm{~B}$, and the execution time of the $1 \mathrm{MHz}$-clock MCU was $3.5 \mathrm{~ms}$.

The NB RF module of the $477 \mathrm{MHz}$ band was tested at a bit rate of $2.4 \mathrm{kbps}$ in a home environment and compared with a wider bandwidth receiver operating at the same rate. The experimental results are illustrated in Figure 16. The transmitter was located on the eighth floor, and the receiver was moved around the space to measure both the RSSI values and the packet error rates (PERs). The narrower bandwidth 


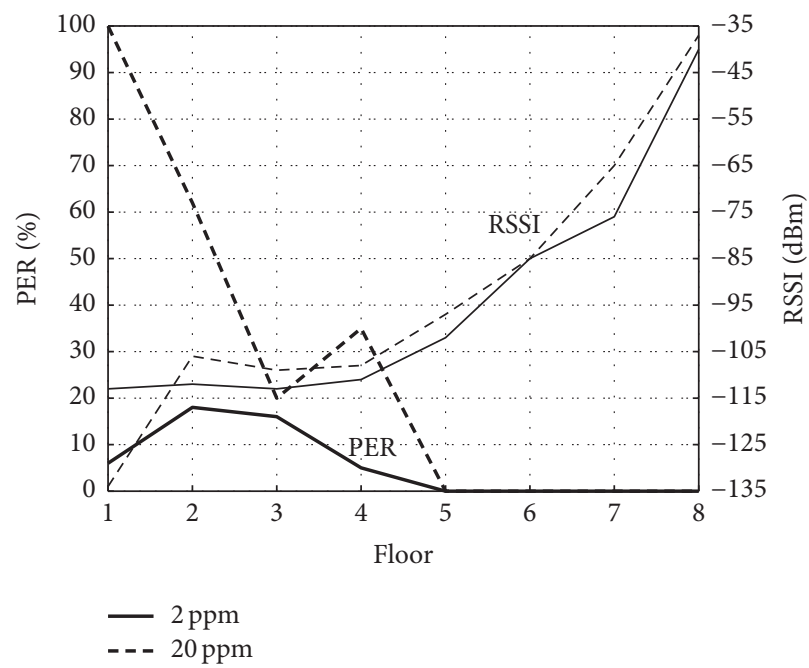

FIGURE 16: Comparison of receiver bandwidths operated under the same bit rate of $2.4 \mathrm{kbps}$ for which the transmitter was located on the eighth floor of the building. The frequency errors of $\pm 2.0 \mathrm{ppm}$ and $\pm 20 \mathrm{ppm}$ had receiver bandwidths of $6.5 \mathrm{kHz}$ and $36.6 \mathrm{kHz}$, respectively.

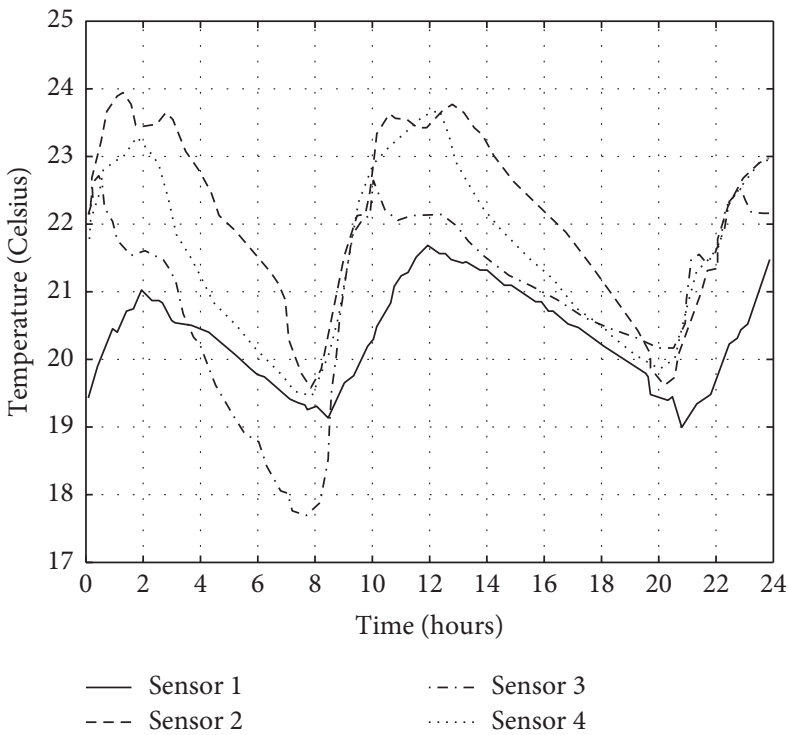

(a)

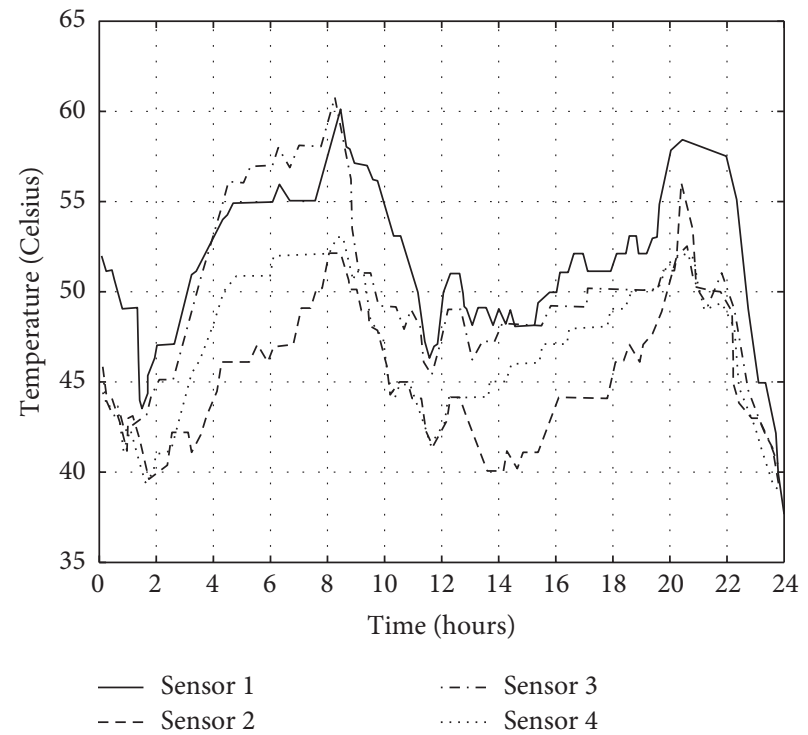

(b)

FiguRE 17: Comparison of receiver bandwidths operated under the same bit rate of $2.4 \mathrm{kbps}$ for which the transmitter was located on the eighth floor of the building. The frequency errors of $\pm 2.0 \mathrm{ppm}$ and $\pm 20 \mathrm{ppm}$ had receiver bandwidths of $6.5 \mathrm{kHz}$ and $36.6 \mathrm{kHz}$, respectively.

receiver demonstrated better packet error rates and could provide longer transmission ranges than the wider bandwidth receiver could. From the results shown in Figure 16, it is clear that the CoAP wireless sensor could cover more than five of the building's stories.

5.2. Experiment Performed on the Hybrid WSN. The proposed hybrid WSN system was constructed in a five-story library building. Each story had one CoAP gateway, two CoAP wireless sensors, and eight simple wireless sensors to collect temperature and humidity data. The data measured in one day, depicted in Figure 17, provided meaningful information with respect to the rooms' locations. In Figure 18, the MOS-based $\mathrm{CO}_{2}$ sensor of IWSM was compared with the conventional NDIR-based $\mathrm{CO}_{2}$ sensors in an office environment with moving people. We can notice that the MOS-based $\mathrm{CO}_{2}$ sensor can efficiently measure the $\mathrm{CO}_{2}$ gas amount change below 2000 ppm similarly to the NDIR-based sensor cases.

\section{Conclusion}

A low-cost hybrid wireless sensor network utilizing the $917 \mathrm{MHz}$ and $447 \mathrm{MHz} \mathrm{RF}$ bands is implemented for indoor 


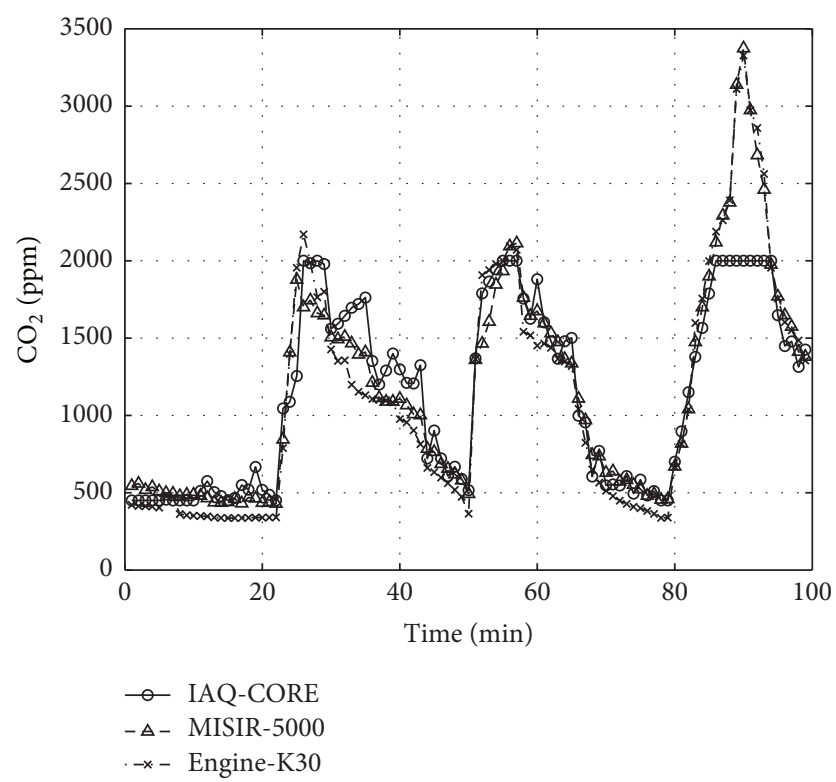

Figure 18: Comparison of the MOS-based $\mathrm{CO}_{2}$ sensor of IWSM (IAQ-CORE) with the conventional $\mathrm{CO}_{2}$ sensors, which are based on NDIR (MISIR-5000 and Engine-K30).

environment monitoring. A duty-cycling receiver algorithm and a digitally controlled temperature-compensation crystal oscillator (DCTCXO) are implemented to achieve lowpower and low-cost wireless sensor nodes. In the proposed DCTCXO, a two-step compensation is performed, in which a fixed third-order curve is applied first and a first-order curve is applied after that, to achieve a high-precision frequency reference. The first-order compensation curve for a given crystal resonator sample is automatically obtained from the frequency-offset measurement conducted in the AFC block. By implementing both the duty-cycling algorithm and the proposed DCTCXO, the average operating current of the RF module is reduced from $16.7 \mathrm{~mA}$ to $4.0 \mathrm{~mA}$.

\section{Conflicts of Interest}

The authors declare that there are no conflicts of interest regarding the publication of this paper.

\section{Acknowledgments}

This work was supported by Korea Institute of Energy Technology Evaluation and Planning (KETEP) and by the Ministry of Trade, Industry \& Energy (MOTIE) of the Republic of Korea (20151210200240 and 20141010501840).

\section{References}

[1] J. Lozano, J. I. Suarez, P. Arroyo, J. M. Ordiales, and F. Alvarez, "Wireless sensor network for in door air quality monitoring," Chemical Engineering Transactions, vol. 30, pp. 319-329, 2012.

[2] S. Bhattacharya, S. Sridevi, and R. Pitchiah, "Indoor air quality monitoring using wireless sensor network," in Proceedings of the 6th International Conference on Sensing Technology (ICST '12), pp. 422-427, IEEE, Kolkata, India, December 2012.

[3] D. S. Kim, S.-Y. Son, and J. Lee, "Developments of the in-home display systems for residential energy monitoring," IEEE Transactions on Consumer Electronics, vol. 59, no. 3, pp. 492-498, 2013.

[4] S. Abraham and X. Li, "A cost-effective wireless sensor network system for indoor air quality monitoring applications," Procedia Computer Science, vol. 34, pp. 165-171, 2014.

[5] T. Torfs, T. Sterken, S. Brebels et al., "Low power wireless sensor network for building monitoring," IEEE Sensors Journal, vol. 13, no. 3, pp. 909-915, 2013.

[6] A. Kim, J. Han, T. Yu, and D. S. Kim, "Hybrid wireless sensor network for building energy management systems based on the 2.4 GHz and $400 \mathrm{MHz}$ bands," Information Systems, vol. 48, pp. 320-326, 2015.

[7] F. Liang and B. Liu, "Research of sub-GHz wireless sensor network and its application in grain monitoring system," Journal of Computational Information Systems, vol. 7, no. 10, pp. 34913498, 2011.

[8] J. Han, J. Lee, E. Lee, and D. S. Kim, "Development of a lowcost indoor environment monitoring system based on a hybrid wireless sensor network," in Proceedings of the IEEE International Conference on Consumer Electronics (ICCE '16), pp. 461462, January 2016.

[9] M. N. Halgamuge, T.-K. Chan, and P. Mendis, "Experiences of deploying an indoor building sensor network," in Proceedings of the 3rd International Conference on Sensor Technologies and Applications (SENSORCOMM '09), pp. 378-381, June 2009.

[10] G. Ghidini and S. K. Das, "An energy-efficient markov chainbased randomized duty cycling scheme for wireless sensor networks," in Proceedings of the 31st International Conference on Distributed Computing Systems (ICDCS '11), pp. 67-76, July 2011.

[11] G. Buroker and M. Frerking, "A Digitally Compensated TCXO," in Proceedings of the 27th Annual Symposium on Frequency Control, pp. 191-198, Cherry Hill, NJ, USA, 1973.

[12] W. Dargie and C. Poellabauer, Fundamentals of Wireless Sensors Networks: Theory and Practice, John Wiley Sons, 2010.

[13] B. Otis and J. Rabaey, "Ultra-low power wireless technologies for sensor networks," Ultra-Low Power Wireless Technologies for Sensor Networks, pp. 1-184, 2007.

[14] A. Mroch and G. Hykes, "A miniature high stability TCXO using digital compensation," in Proceedings of the 30th Annual Symposium on Frequency Control, pp. 292-300, 1976.

[15] J. C. Blanco, F. J. Azcondo, and J. Peire, "New Digital Compensation Technique for the Design of a Microcomputer Compensated Crystal Oscillator," IEEE Transactions on Industrial Electronics, vol. 42, no. 3, pp. 307-315, 1995.

[16] Q. Huang and P. Basedau, "Design considerations for highfrequency crystal oscillators digitally trimmable to sub-ppm accuracy," IEEE Transactions on Very Large Scale Integration (VLSI) Systems, vol. 5, no. 4, pp. 408-416, 1997.

[17] D. S. Kim, E. Lee, J. Han, and J. Lee, "Digitally controlled temperature-compensated crystal oscillator in developing lowcost radio frequency modules," Information, vol. 18, no. 7, pp. 3157-3166, 2016.

[18] R. M. Cerda, Understanding Quartz Crystals and Oscillators, Artech House Microwave Library, London, UK, 2014. 


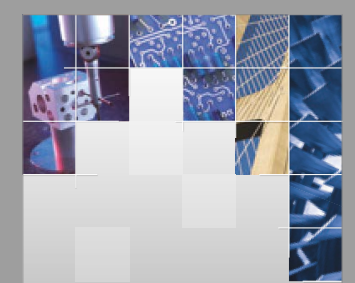

\section{Enfincering}
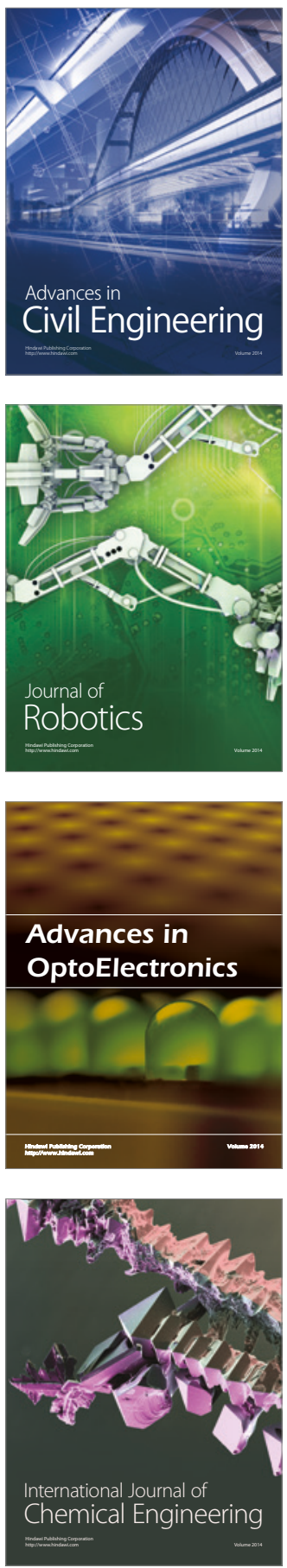

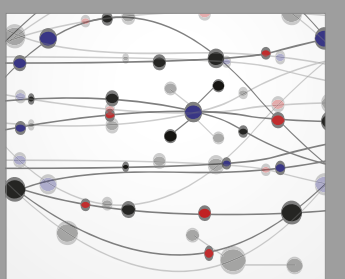

The Scientific World Journal

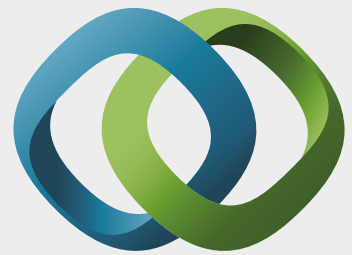

\section{Hindawi}

Submit your manuscripts at

https://www.hindawi.com
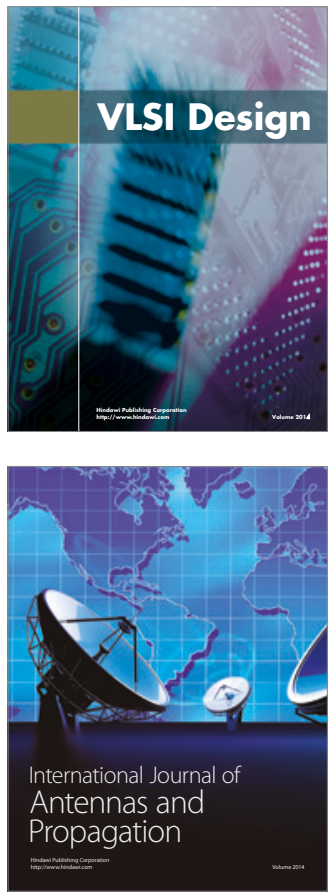

\section{Rotating}

Machinery
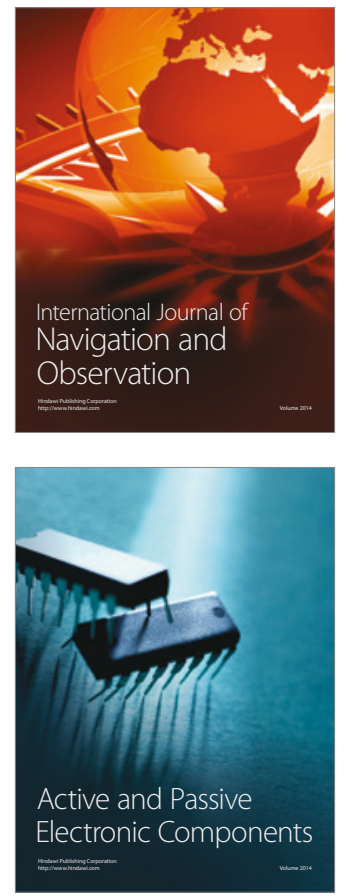
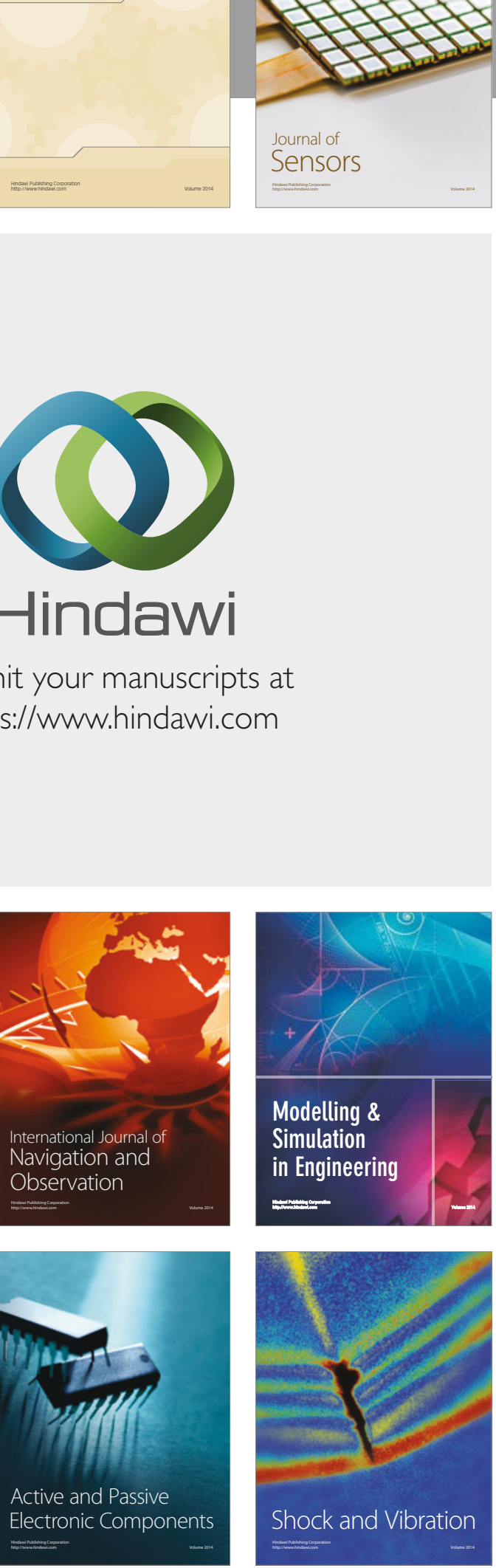
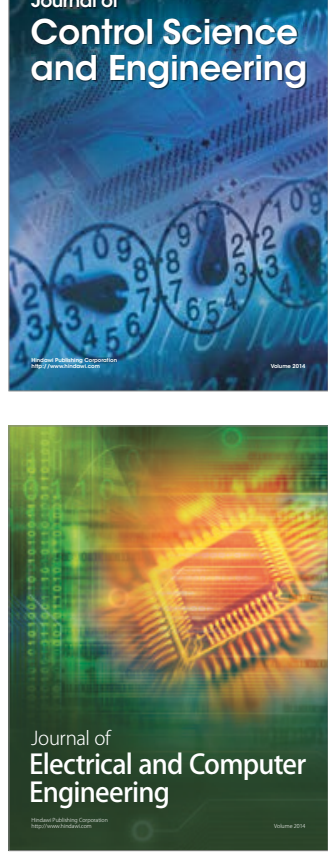

Distributed

Journal of

Control Science

and Engineering
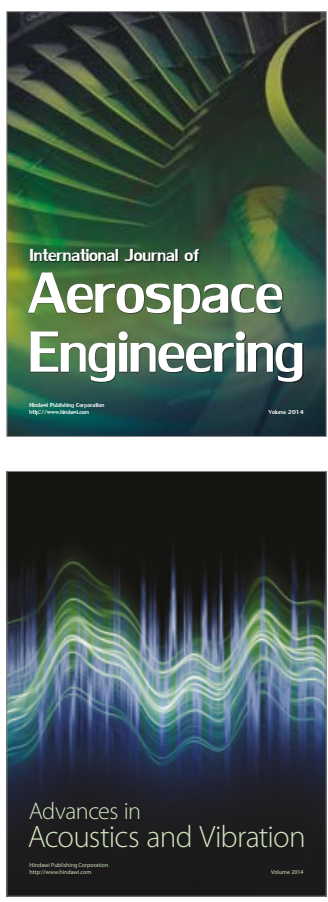

Sensor Networks 